\title{
Facilitators and Barriers to Fitness Bootcamp Participation Using the Behaviour Change Wheel
}

Leo De Winter and Leslie Morrison Gutman

Department of Clinical, Educational and Health Psychology, UCL, London UK

De Winter, L., \& Gutman, L. M. (2021). Facilitators and barriers to fitness bootcamp participation using the Behaviour Change Wheel. Health Education Journal, 00178969211044180.

\begin{abstract}
Objective: Despite its importance for mental and physical health, many adults fail to meet current physical activity recommendations. Furthermore, most adults who begin a physical activity programme revert to being less active or even inactive within the first six months. Fitness bootcamps represent a potential intervention for improving physical activity in healthy adults. However, no study to date has examined their influences on long-term participation, which is the first step in developing an effective intervention. Using the Behaviour Change Wheel (BCW) framework, this study identified the facilitators and barriers to long-term (1 year or more) bootcamp participation, which were then linked to behaviour change techniques (BCTs) to facilitate intervention development.

Design: Qualitative research design.

Setting: Data collection occurred in London, United Kingdom.

Method: Interviews were conducted with 15 long-term fitness bootcamp participants.

Results: Thematic analysis revealed 17 facilitators and 6 barriers to long-term bootcamp participation. Participants highlighted the importance of facilitators such as convenience, being outdoors and enjoyment. The social environment, including having a positive instructor and supportive group members, was further noted as a key influence on participation. While long-term bootcamp participants faced occasional barriers to their routine such as physical injury, bad weather and competing events, the impact of these could be minimised through appropriate BCTs such as 'demonstration of the behaviour' and 'restructuring the social/physical environment'.

Conclusion: Findings from this study highlight the importance of social and environmental factors to promoting long-term fitness bootcamp participation and the inclusion of social and physical environmental restructuring as key intervention components.
\end{abstract}

Keywords: Physical Activity Maintenance; Fitness Bootcamp Participation; Behaviour Change Wheel; Behaviour Change Techniques; Intervention Design

\section{Corresponding author:}

Leslie Morrison Gutman, Department of Clinical, Educational and Health Psychology, UCL, London, 1-19 Torrington Place, London WC1E 7HB, UK

Email: I.gutman@ucl.ac.uk 


\section{Introduction}

Physical inactivity is associated with higher rates of non-communicable disease including coronary heart disease, colon cancer, hypertension, depression and anxiety (Lee et al., 2012; Rebar et al., 2015). Despite the importance of physical activity for mental and physical health, 33\% of UK adults fail to meet the current recommendations of at least 75 minutes of vigorous activity per week, 150 minutes of moderate intensity or an equivalent combination of the two (WHO, 2018). In addition, most of those who begin a physical activity programme revert to being less active or even inactive within the first six months (Marcus et al., 2000). With this in mind, an understanding of the influences on physical activity maintenance is an essential first step in the design of interventions to promote long-term behaviour change (Rhodes \& Pfaeffli, 2010).

Fitness bootcamps represent a potential intervention for improving physical activity in healthy adults. Fitness bootcamps are a physical training programme led and organised by a professional instructor which occur at a fixed place and time. Classes often take place outdoors and participants are normally asked to engage in a variety of different activities. Fitness bootcamps have grown in popularity; however, no study to date has examined the influences on long-term bootcamp participation, especially using a systematic and validated tool for the design and evaluation of behaviour change interventions. Using the Behaviour Change Wheel (BCW) framework, the present study conducted qualitative interviews with long-term (1 year or more) bootcamp participants to identify the facilitators and barriers to their participation, which were then linked to intervention components in order to facilitate intervention development (Michie, Atkins, \& West, 2014).

\section{Behaviour Change Wheel Framework}

The BCW is a systematic, validated theoretical framework developed from 19 key frameworks of behaviour change (Michie et al., 2014). The BCW involves a step-by-step process for the design of behaviour change interventions (Michie et al., 2014). The COM-B model of behaviour constitutes the core of the BCW and can be used to facilitate the first steps of intervention design, which focus on understanding the facilitators and barriers to a target behaviour (Michie, Atkins, \& West, 2014). The COM-B model states that capability, opportunity and motivation interact to produce behaviour. As shown in Figure 1, these three domains influence whether the behaviour is performed and performing the behaviour can, in turn, influence these domains. Capability and opportunity can further influence motivation. Each of the three domains can be split into two sub-domains (see Figure 1). The next layer in the BCW identifies 9 intervention functions that can be selected based on the facilitators and barriers identified in the COM-B diagnosis. The final layer of the BCW identifies seven types of policy functions that can be used to support the delivery of intervention functions.

Figure 1 about here

The Behaviour Change Technique (BCT) Taxonomy (BCTT v1) collates a list of 93 evidence-based BCTs that form the intervention components or 'active ingredients' of behaviour change interventions. Expert consensus allows for mapping the COM-B domains to broader intervention functions on the $\mathrm{BCW}$, which are then linked to appropriate BCTs to 
produce behaviour change (Michie et al., 2014). Using this mapping, facilitators and barriers can be linked to BCTs that, when applied, could produce behaviour change.

The BCW framework offers an evidenced-based approach to behaviour change, encouraging intervention designers to consider a full range of options and choose those that are the most promising. The framework has been successfully used to develop physical activity interventions, such as increasing physical activity advice given by healthcare professionals to cancer patients (Murtagh et al., 2018). The COM-B model has further been shown to be valid measure of the influences on physical activity (Howlett et al., 2017) and the BCTT has also been used in systematic reviews of physical activity interventions to identify effective BCTs for maintenance (Howlett, Trivedi, Troop, \& Chater, 2019; Murray et al., 2017).

\section{Physical Activity Interventions}

Numerous reviews have shown that behaviour change interventions can promote physical activity (e.g., McEwan et al., 2016; Williams \& French, 2011). Physical activity maintenance in behaviour change interventions, however, has received somewhat less attention. One reason for this is that physical activity levels are most often measured immediately after intervention completion (Bull et al., 2014; Rhodes et al., 2017). One systematic and metaanalytic review, for example, found that less than one in four physical activity intervention studies reported outcomes longer than 3-12 months post-baseline (Bull et al., 2014). Several reviews have further concluded that physical activity interventions show weak or no effects on maintenance (Howlett et al., 2019; Murray et al., 2017). In terms of intervention strategies, prompting practice of the behaviour and increasing the intensity and duration of exercise in progressive stages were found to be effective intervention strategies in producing longer term changes in physical activity (Howlett et al., 2019).

These reviews underline that additional research is needed to understand the specific influences on physical activity maintenance. This is an important first step to intervention design to produce lasting behaviour change. It is suggested that this type of analysis should be context-specific, focusing on a behaviour in a specific setting (Michie et al., 2014). Given the unique context and setting of fitness bootcamps in comparison to other modes of physical activity, an examination of the influences on long-term maintenance can contribute to the potential design of interventions in this context.

\section{Present Study}

Using the BCW framework, the present study first explores facilitators and barriers to physical activity maintenance among long-term fitness bootcamp participants. Using expert mapping informed by the BCW approach, the study further identifies BCTs for the purposes of intervention development. To improve reporting quality, recommendations from the Standards for Reporting Qualitative Studies are followed (SRQS; O'Brien et al., 2014).

\section{Methods}

\section{Participants}


Convenience sampling was used, facilitated by the assistance of three instructors of fitness bootcamp groups. People qualified as potential participants if they met the inclusion criteria. The inclusion criteria were: (1) has been part of a bootcamp group for at least 1 year; (2) identified bootcamps as their primary physical activity; (3) has been meeting the UK physical activity guidelines for the last year (12 months) in terms of at least 75 minutes of vigorous physical activity per week, 150 minutes of moderate intensity physical activity or an equivalent combination of the two (CMO, 2011); and (4) aged 19-64 years. An age limit was defined because UK physical activity guidelines are specific to a certain age group (Department of Health and Social Care, 2011).

\section{Materials}

An interview schedule was developed to promote consistency across interviews, consisting of 13 questions with a range of more specific prompts. The COM-B model was used to develop the questions and prompts were developed using a review of theoretical explanations of behavioural maintenance (Kwasnicka et al., 2016). Example questions and prompts included 'How does your knowledge about fitness influence whether you exercise?' (psychological capability); 'How does your physical stamina and strength influence whether you exercise?' (physical capability); 'How does the physical environment influence how much you exercise?' (physical opportunity); 'How do the people around you influence how much you exercise?' (social opportunity); 'What really motivates you to maintain this level of exercise?' (automatic/reflective motivation); and 'What are your current exercise goals?' (reflective motivation).

\section{Procedure}

Instructors from three different fitness bootcamp groups invited members of their group to participate in the study via text message. If potential participants told their instructors they would like to participate, they were then contacted via e-mail by the first author with a participant information sheet and consent form. The majority of interviews took place inperson $(n=10)$, but if an in-person interview was deemed inconvenient by participants, then it was conducted via Skype $(n=3)$ or over the phone $(n=2)$. Recent evidence suggests that differences between in-person and video interviews are modest (Krouwel, Jolly, \& Greenfield, 2019). All interviews were conducted by the first author and lasted 60 minutes on average. Participants were sent a GBP 7.50 gift voucher after the interview. All interviews were recorded and transcribed verbatim by the first author, and all references to places and people were replaced by pseudonyms. Data saturation was reached, which was seen as characterised by 'no new data, no new themes, no new coding' (Fusch \& Ness, 2015, p. 1409).

\section{Data Analysis}

Thematic analysis was used to identify the facilitators and barriers to physical activity maintenance. Braun, Clarke and Weate's (2016) overview of thematic analysis for sport and exercise research was flexibly applied to develop the analysis plan. Their 'flexible' approach to thematic analysis offers robust processes for identifying patterns, and interpreting them, in a number of different ways including deductive and inductive coding. In practice, most 
thematic analysis includes both deductive and inductive elements (Braun et al., 2016). In line with other qualitative studies using the BCW (e.g. Moran \& Gutman, 2021), this study used a deductive then inductive approach. This entailed an initial coding of the interview data based on the COM-B model, followed by the identification of data-driven sub-themes within each domain to provide a more in-depth understanding of the influences on behaviour.

During phases 1 and 2 (data familiarisation and coding), the first author (LD) transcribed the interviews verbatim and then read and re-read the transcripts to become immersed in the data. Using the COM-B model, LD first coded the transcripts based on the deductive approach. This was followed by generating sub-themes within each COM-B domain using an inductive approach. Each sub-theme was 'tagged' to represent a specific barrier or facilitator to physical activity maintenance.

Phases 3-5 (theme development, refinement and naming) involved reviewing and revising the sub-themes and developing an analysis of the data represented by the subthemes. First, LD checked the sub-themes against original data quotes to ensure they robustly represented the COM-B domain. Then, a second person coded several of the transcripts independently to facilitate refinement of the themes and ensure consistency in their structure and content. Any disagreements were resolved through discussion and further refinement of the sub-themes. After this process, the sub-themes re-checked with the data extracts to ensure they were correctly represented, with any further refinement undertaken as necessary.

Lastly, the second author (LG) identified corresponding intervention functions and $\mathrm{BCT}$, based on the COM-B facilitators and barriers. First, the COM-B $\times B C W$ intervention function matrix was employed to identify effective intervention functions for each COM-B barrier and facilitator (Michie et al, 2014). Then, possible behaviour change techniques were identified, based on previous guidance from expert consensus (Michie et al., 2014). Using the APEASE criteria (Affordability, Practicability, Effectiveness and cost-effectiveness, Acceptability, Safety and side-effects and Equity), BCTs were identified based on the suitability of their implementation in this context (Michie et al, 2014).

\section{Ethical Considerations}

Ethical approval for this study was given by the UCL Research Ethics Committee (Project ID: 15105/001).

\section{Results}

Fifteen participants were recruited. Table 1 provides an overview of participant characteristics. The sample consisted mostly of women $(n=13)$, with a median age of 50.5 years. The median length of bootcamp membership was 2.5 years. Each bootcamp (BC) group was led by a personal trainer (PT), with $\mathrm{BC} 1$ being led by PT1, BC2 by PT2 and BC3 by PT3. The trainers of $\mathrm{BC} 1, \mathrm{BC} 2$ and $\mathrm{BC} 3$ estimated the proportion of women in their group to be $61 \%, 100 \%$ and $90 \%$, respectively, with the average length of membership being around 2.5 years, 3 months and 3.5 years respectively.

Table 1 about here 
A total of 17 facilitators and 6 barriers were identified (see Table 2). Facilitators included convenience, a positive instructor, health benefits, enjoyment and other themes. The identified barriers consisted of events that can temporarily disrupt the exercise routine (e.g., physical injury, bad weather, travel) and participants highlighted several facilitators that minimised the impact of such disruptions (e.g., prioritising bootcamp participation, upfront payment, multiple sessions).

Table 2 about here

\section{Psychological Capability}

\section{Increased Exercise Knowledge (facilitator)}

Participants explained how exercise knowledge enabled their bootcamp participation even when disruptions occurred. For example, in case of injury: 'Yeah, a knee operation, but ... having trained for so long, and having been to all classes, I know how to modify a lot of the things... which is why I have never stopped' (P2, F, age: 55, BC: 5 years). This quote illustrates that knowledge may result from previous participation in bootcamp classes and can help to minimise the impact of barriers such as physical injury and illness.

\section{Physical Capability}

\section{Physical Injury (barrier)}

Injury can disrupt people's routine by preventing them from doing the type of activity that they usually do. For example, one participant said: 'When I was younger, I hurt my knee ... Sometimes I can push through it, sometimes I ... have to get the instructor to work out a different routine for me' (P1, F, age: 39, BC: 3 years). Injury does not necessarily prevent one from going to bootcamp, since participants are asked to do a range of different activities in every bootcamp, and instructors can change the routine (see sub-theme 'Instructor').

\section{Physical Illness (barrier)}

Illness can sometimes prevent participants from going to the bootcamp session. Even participants who rarely missed a session described illness as a barrier: 'I mean, if I'm ill, I won't go. But otherwise, I'll go' (P12, F, age: 63, BC: 5 years) and 'Unless I am unwell... That would be the only time that I would not go and train' (P2, F, age: 55, BC: 5 years).

\section{Physical Opportunity}

\section{Convenience (facilitator)}

Nearly every participant commented on how bootcamp participation 'fitted' with their other activities, such as work commitments and family life. The location of the session seemed particularly important. Bootcamps were usually close to home, or on the way home from work. One participant said, for example, '[bootcamp]'s really convenient to get to, it's 
5 minutes from my house by bike ... I'm a member of ... [Name of health club] ... I never go, because it's further away' (P13, F, age: 50, BC: 1 year). Several participants also spoke about the bootcamp happening at a convenient time. For example: 'I have to fit it around my schedule .... That is one of the other reasons why I choose to do it in the mornings ... it is usually mornings or nothing' (P3, M, age: $50, B C: 2$ years).

\section{Being Outdoors (facilitator)}

The majority of participants valued the fact that the bootcamp occurred outside. For instance: 'I much prefer being outside. I have no desire to be exercising in a gym. It just doesn't hold any appeal to me at all. So being outside is brilliant' (P8, F, age: 52, BC: 2.5 years).

\section{Variety of Activities (facilitator)}

Participants appreciated that the bootcamp involved a variety of different activities and the content varies over time. For example: 'I enjoy the variety. Because anything I do myself tends to be very much the same' (P8, F, age: $52, B C: 2.5$ years).

\section{Competing events (barrier)}

Competing events occasionally prevented people from going to the bootcamp, as the following quote demonstrates: 'sometimes the time restrictions make it hard ... sometimes you wouldn't be able to make it' (P15, F, age: 33, BC: 2 years).

\section{Bad weather (barrier)}

Bad weather was another reason for participants to miss a bootcamp session. For example: 'I don't like coming out in, I don't like going to bootcamp in the rain. And that sort of risk of getting cold and wet and risking catching a cold or something' (P7, F, age: 51, 6 years).

\section{Travel (barrier)}

Travel could disrupt participants' routine. If participants were out of town, they could not attend the bootcamp, for instance: 'I do go back and forth to [country name] quite a bit, so I will be gone for, you know, a week, sometimes two, and so those, those are the times that I miss' (P11, F, age: 58, BC: 6 years).

\section{Upfront payment (facilitator)}

Disruptions did not always stop participants' attendance. Participants reported that paying upfront for the bootcamp sessions promoted attendance when faced with disruptions. For example: 'I pay upfront, so I've already paid. If you're paying a drop-in rate, and it's pouring with rain, you might think you'll save your 10 quid and not go, whereas I've paid, so I just go' (P8, F, age: 52, BC: 2.5 years).

\section{Multiple Sessions (facilitator)}


Participants sometimes missed a session due to unexpected events, and there were also cases where a change in people's life (e.g., children going to school, changing jobs) made it impossible to attend the bootcamp at their usual time and/or day. The availability of multiple sessions allowed people to either make up for a missed session or keep attending bootcamps, for instance: 'I do my absolute best to get to those [bootcamps] on a Tuesday night every week, and usually if I miss the Tuesday one, I will pick up on a Saturday morning [when there is also a bootcamp, that P4 normally doesn't go to]' (P4, M, age: 28, BC: 18 months).

\section{Social Opportunity}

\section{Positive Instructor (facilitator)}

Almost all the participants described the instructor as a positive influence. There were multiple ways in which an instructor could influence attendance and physical activity maintenance. First, nearly all participants described how a good instructor could make the bootcamp more fun. It seemed critically important that an instructor was engaging, for example: 'the guy that takes the Saturday and the, the Monday and the Saturday sessions, his sessions are always booked ... he's really kind of like, I don't know, he gets you going ... the delivery of the message by the personal trainer and their personality are really important' (P13, F, age: 50, BC: 1 year). Second, half the participants also described how instructors adapted the routine, which allowed them to keep doing physical activity despite injury or chronic health issues: 'I do have like, carpal tunnel in my wrists ... and that's why I think a good trainer is important, because they can adapt things for you' (P15, F, age: 33, BC: 2 years).

\section{Group Members (facilitator)}

Participants noted that the social element had a positive influence on their participation, for example: 'you see the same people there quite consistently and we have made a couple of really-really good friends just from that ... the social element of that has been really positive, and that is one of the big reasons why we've kept on with it rather than trying something else' (P4, M, age: 28, BC: 18 months).

\section{Supportive Partner (facilitator)}

Participant's partners were another facilitator in the social environment that seemed to influence physical activity maintenance. Some people reported that their partner engaged in physical activity on a regular basis which seemed to set an example or establish a social norm, for example: 'My husband is a real exerciser, so I can't, I would then feel guilty if he does all this and he's super fit, and I, and he has a wife that isn't doing anything' (P10, F, age: 55, BC: 7 years). Participants also reported that partners can facilitate maintenance by actively stimulating them to go, for instance: 'if I wake up on a Saturday morning and I am really tired because I've had a long week, and I missed the HIIT session on a Tuesday, she will be the one to say: '[P4], get out of bed. You should go to HIIT' (P4, M, age: 28, BC: 18 months). 


\section{Reflective Motivation}

\section{Health Benefits (facilitator)}

Health benefits were mentioned by nearly every participant as a reason to keep doing physical activity, for instance: 'everyone is supposed to be exercising, it's proven this, that and the other. It helps with diabetes, blood pressure, uhm, everything like that. So, I should be doing, everybody, kind of what you read, what you're told, you must do this. You must, it's almost like: 'OK... I'll do it" (P10, F, age: 55, BC: 7 years). Interestingly, many participants made a link between physical activity and old age when talking about the health benefits of regular physical activity, for example: 'what motivates me is the health benefits of exercise. And knowing that it will hopefully lead me into old age that I am still able to function properly' (P2, F, age: 55, BC: 5 years).

\section{Weight loss/maintenance (facilitator)}

Another common motivation for bootcamp participation was losing and maintaining weight. The emotional intensity of quotes was generally high, and participants recognised this as a very important motivator, for example: 'If I was to rank them, the number one is my weight.... So that is my primary motivator' (P13, F, age: 50, BC: 1 year) and 'I often do have issues with my weight .... And I know it is one of those things that if I don't do exercise, it could flip quite easy' (P4, $\mathrm{M}$, age: $28, \mathrm{BC}$ : 18 months).

\section{Prioritising sessions (facilitator)}

Participants repeatedly described making a deliberate effort to prevent events from competing with their bootcamp sessions. The reduced the possible influence of competing events, as described in this quote: 'if I'm making doctor's appointments, or dentist appointments, I will try to avoid that time [time of the bootcamp]' (P11, F, age: 58, BC: 6 years).

\section{Exercise Goals (facilitator)}

Having explicit exercise goals was only mentioned by a few participants from $B C 2$. The interviews showed that the approach to training was different in $\mathrm{BC} 2$ compared to $\mathrm{BC} 1$ and $\mathrm{BC} 3$, because the PT in $\mathrm{BC} 2$ sat down with participants on a regular basis to define goals and monitor progress, for example: '[PT] is very keen on having a goal ... he will get you to set your own personal goals for the week ..., and then at the end of the week you have to check in with him and say: 'OK, against those goals, how did you do?' In contrast, concrete goals were not mentioned by participants from BC1 and BC3. Instead, these participants described previously having concrete goals, but now being more focused on enjoyment: ‘ used to [have exercise goals] ... I used to be a case of 'All right, ... I need to be able to run $10 \mathrm{k}$ in a certain amount of time', or something like that, whereas now I don't, now I just ... do it for the enjoyment of it' (P4, M, age: 28, BC: 18 months). Interestingly, the BC2 participants suggested that setting and chasing goals can harm enjoyment: 'I have probably overdosed a bit on fitness ... training for [goal] was really building up a lot of protein, doing a 
lot of strength training, and endurance training ... and I lost motivation' (P6, F, age: not disclosed, BC: 13 months).

\section{Accountability (facilitator)}

Accountability to others in the group was seen as a facilitator. When asked if there were any things that made it easier to maintain the schedule, one participant's immediate response was: 'Accountability. Without any doubt, without any hesitation, it's accountability' (P6, F, age: not disclosed, BC: 13 months). Others expressed similar sentiments: 'Having a group of people that kind of hold you accountable to show up ... that's a huge benefit' (P15, F, age: 33, BC: 2 years).

\section{Social Identity (facilitator)}

Several people described bootcamp and/or sports participation as a key part of their social identity: 'It is, it is just part of me, [P10] does bootcamp' (P10, F, age: 55, BC: 7 years) and 'I love most sports ... it is a very big part of who I am' (P4, M, age: 28, BC: 18 months).

\section{Automatic Motivation}

\section{Enjoyment (facilitator)}

Enjoyment was mentioned by nearly every participant and people described it as a very important reason to maintain the schedule, for example, 'Enjoyment is my main reason. I do love the classes.' (P1, F, age: 39, BC: 3 years) and 'I want to keep my body fit enough ... but that is not as much a motivation as the enjoyment' (P13, F, age: 50, BC: 1 year).

\section{Feeling Good (facilitator)}

Feeling good was mentioned by most participants. More specifically, participants described always feeling better after the bootcamp sessions: 'I always feel better afterwards ... it [bootcamp] makes you feel better and energized' (P3, M, age: 50, BC: 2 years) and '[bootcamp]'s a mood enhancer. I think you always feel better after you've done it' (P11, F, age: 58, BC: 6 years).

\section{Stress (barrier)}

Stress was identified as a sub-theme because a few participants described it as the most common reason to miss a session, for example: 'my job is, can be quite stressful at times. And sometimes I think: 'Ouch. [exhausted sigh], I just can't think straight' ... my body might want to train, but I am just not in the right mindset to do it.' (P2, F, age: 55, BC: 5 years).

\section{Behaviour Change Techniques}

Given the identified facilitators and barriers, the BCTs of 'restructuring the physical or social environment', 'demonstration of the behaviour' and 'instruction on how to perform the behaviour' are suggested for long-term bootcamp participation. 'Restructuring the physical 
or social environment', which involves changing the physical or social contexts to facilitate or remove barriers to the desired behaviour, aligns with facilitators such as convenience/being outdoors and social group/positive instructor, respectively, and physical barriers such as bad weather and competing events. 'Demonstration of the behaviour', which involves providing an observable example of the behaviour for the person to copy and aspire to, corresponds to facilitators such as feeling good and enjoyment. Lastly, 'instruction on how to perform the behaviour', which involves advising the person on how to perform the behaviour, corresponds to the facilitator of increased exercise knowledge as well as barriers such as physical injury or illness.

\section{Discussion}

This study has explored facilitators and barriers to long-term participation in fitness bootcamps, which are linked to corresponding BCTs for the purposes of physical activity intervention design. Twenty-three facilitators and barriers ( 17 facilitators and 6 barriers) were identified across the sub-domains of the COM-B model. Corresponding BCTs addressing facilitators and barriers are explained below.

\section{Facilitators and barriers}

Key facilitators of fitness bootcamp participation included convenience and the outdoor setting. Other research has also found that convenience (in terms of distance to the health and fitness venue) is associated with longer physical activity behaviour (Rand et al., 2019). The importance of convenience implies that bootcamps should be in an opportune location and take place at useful times for the participants. The most convenient location and time depends on the commitments and activities of individual participants. Other studies have highlighted the outdoors as a key facilitator of physical activity (e.g., Lesser, Nienhuis, \& Belanger, 2021). With this in mind, 'restructuring the physical environment', such as offering outdoor activities at different times and locations, may be effective in promoting physical activity maintenance in fitness bootcamps.

Findings also showed that enjoyment and feeling good facilitate physical activity maintenance in fitness bootcamps. This is in line with previous studies which found that motivation (i.e., enjoyment) was associated with maintenance of sporting activities (Knittle et al., 2016; Williams et al., 2006). However, these previous studies only looked at maintenance over a period of 4-6 months. The findings from the present study suggest that the relationship between motivation and physical activity maintenance also exists when a longer time cut-off (i.e., at least 1 year) defines maintenance for bootcamp participation. A corresponding BCT is 'demonstration of the behaviour', which might involve having an instructor and/or group members model enjoyment and enthusiasm during bootcamp participation.

The findings further highlight the social influences on physical activity maintenance, especially for fitness bootcamp participants. This is not surprising given the prominence of the instructor and the group. This aligns with previous studies suggesting group membership facilitates physical activity maintenance (Crossley, 2006; Kwasnicka et al., 2016). Interestingly, systematic reviews have not found social support to be a significant component of maintenance in physical activity interventions (i.e., Murray et al., 2017). Rather than emphasising practical or emotional social support, our findings suggest that 
social influences from their instructor, partner or fellow group members are expressed in terms of accountability to the group, social norms of physical activity and positive affect. This is in line with the COM-B model, which shows that social opportunities can increase motivation which, in turn, influences behaviour. With this in mind, 'restructuring the social environment' to facilitate increased contact and time spent with the instructor and among group members appears to be an important component of physical activity maintenance in fitness bootcamps.

A few barriers were mentioned regarding long-term fitness bootcamp participation, which represent temporary disruptions to the routine. Even long-term bootcamp participants faced disruptions (e.g., bad weather, competing events, physical injury). However, facilitators can minimise the impact of such disruptions. With this in mind, 'restructuring the physical environment', such as offering multiple sessions per week, encouraging participants to pay upfront for a certain number of classes per week and adapting activities based on people's capabilities, may be an appropriate BCT. 'Instruction on how to perform the behaviour' may also be effective in terms of imparting knowledge on how to adapt their physical activity when dealing with barriers such as physical injury or illness.

Interestingly, the findings also highlight a relatively lack of importance for setting exercise goals for long-term fitness bootcamp participants. This diverges from previous review studies which have concluded that a focus on defining goals and monitoring progress makes physical activity interventions more effective (Knittle et al., 2018; McEwan et al., 2016). Since existing evidence is mostly based on measurements of short-term effectiveness (Bull et al., 2014; Rhodes et al., 2017), it may be that a focus on goals effectively promotes short-term change but is not an effective strategy for longer term maintenance, especially for fitness bootcamp participants. However, more general goals, such as improving health and maintaining weight loss, were frequently mentioned by participants. This may reflect that, while many participants worked to maintain their fitness goals, they were more focused on intrinsic and social reasons (e.g., enjoyment and accountability to the group) for their continued bootcamp participation. This further suggests that fitness bootcamp interventions should ensure that instructors emphasise and model intrinsic reasons for participation, once participants reach their more explicit goals.

\section{Limitations}

The present study was an exploratory one and therefore findings should be interpreted in this context. Given the focus on fitness bootcamps, reported facilitators and barriers are likely to be specific to long-term bootcamp participation. A preference for a group-based, outdoor activities with a dynamic instructor might well be some of the reasons people initially joined a bootcamp, and still may be the reasons they continue to engage in bootcamps as their preferences have not changed. Furthermore, all of the participants were long-term bootcamp participants, which limits the extent to which the findings can be used to facilitate the transition from active participation to long-term maintenance. As this transition has a risk of activity participation regression, future research should examine participants who have yet to reach the maintenance phase, which could better identify strategies to support the maintenance phase transition. Moreover, BC1 and BC3 were both in London and facilitators such as a convenient location may be more important in a large city than in a small town. Lastly, the sample was predominantly women, which was 
reflective of these particular bootcamp groups but not necessarily bootcamps, more generally. Additionally, data on ethnicity was not collected from participants. These limitations likely reduce the generalisability of the findings.

\section{Implications and Conclusion}

The present study explored facilitators and barriers to long-term fitness bootcamp participation and identified corresponding BCTs to produce behaviour change, which represent the first steps in intervention design using the BCW.

Interventions may consider restructuring the physical and social environment to encourage long-term maintenance, as well as possible initial short-term engagement, in fitness bootcamps. In terms of the social environment, this might entail having an enthusiastic instructor and a consistent group of supportive fellow members, with some level of accountability for continued participation. The physical environment can also be restructured to facilitate ease of participation and minimize occasional yet unpredictable disruptions, for example, by offering multiple sessions at a convenient location. In line with COM-B, both the physical and social environment also seem to increase motivation to participate, such as through having a variety of activities in an outdoor setting and increasing enjoyment with an engaging and fun instructor.

In conclusion, findings from this study highlight key influences on long-term participation in fitness bootcamps for healthy adults, emphasising the importance of the social and physical environment. Future studies should explore whether the findings are applicable to other types of outdoor activities particularly those in social settings, such as walking and biking groups.

\section{Acknowledgements}

James Hardy helped conceptualise the study and provided key contacts for obtaining participants. Anna Clifford was the second coder of the interviews for the purpose of establishing reliability.

\section{Statement of Competing Interests}

The authors have no competing interests. 


\section{References}

Braun V, Clarke V and Weate P (2016) Using thematic analysis in sport and exercise research. Routledge Handbook of Qualitative Research in Sport and Exercise Sep(13): 191-205.

Bull ER, Dombrowski SU, McCleary N and Johnston M (2014) Are interventions for lowincome groups effective in changing healthy eating, physical activity and smoking behaviours? A systematic review and meta-analysis. BMJ Open 4(11): p.e006046.

Chief Medical Officers 2019 Physical activity guidelines for adults (19-64 years). Available at: https://assets.publishing.service.gov.uk/government/uploads/system/uploads/attach ment data/file/832868/uk-chief-medical-officers-physical-activity-guidelines.pdf (accessed on 09 August 2021).

Crossley N (2006) In the gym: Motives, meaning and moral careers. Body \& Society 12(3): 23-50.

Fusch, PI and Ness LR (2015) Are we there yet? Data saturation in qualitative research. The Qualitative Report 20(9): 1408-1416.

Howlett N, Trivedi D, Troop NA and Chater AM (2019) Are physical activity interventions for healthy inactive adults effective in promoting behavior change and maintenance, and which behavior change techniques are effective? A systematic review and metaanalysis. Translational Behavioral Medicine 9(1): 147-57.

Knittle K, De Gucht V, Hurkmans E, Vlieland TV and Maes S (2016) Explaining physical activity maintenance after a theory-based intervention among patients with rheumatoid arthritis: Process evaluation of a randomized controlled trial. Arthritis Care \& Research 68(2): 203-10.

Knittle K, Nurmi J, Crutzen R, Hankonen N, Beattie M and Dombrowski SU (2018) How can interventions increase motivation for physical activity? A systematic review and metaanalysis. Health Psychology Review 12(3): 211-30.

Krouwel M, Jolly K and Greenfield S (2019) Comparing Skype (video calling) and in-person qualitative interview modes in a study of people with irritable bowel syndrome-an exploratory comparative analysis. BMC Medical Research Methodology 19(1): 1-9.

Kwasnicka D, Dombrowski SU, White M and Sniehotta F (2016) Theoretical explanations for maintenance of behaviour change: a systematic review of behaviour theories. Health Psychology Review 10(3): 277-96.

Lee IM, Shiroma EJ, Lobelo F, Puska P, Blair SN and Katzmarzyk PT (2012) Lancet Physical Activity Series Working Group. Effect of physical inactivity on major noncommunicable diseases worldwide: an analysis of burden of disease and life expectancy. The Lancet 380(9838): 219-29

Lesser IA, Nienhuis CP, and Belanger L (2021) Active by nature: exploring cancer survivors' exercise barriers, facilitators, preferences, and psychosocial benefits of engaging in outdoor physical activity. Supportive Care in Cancer 29(7): 4095-4103.

Marcus BH, Forsyth LH, Stone EJ, Dubbert PM, McKenzie TL, Dunn AL and Blair SN (2000) Physical activity behavior change: issues in adoption and maintenance. Health Psychology 19(1S): 32.

McEwan D, Harden SM, Zumbo BD, Sylvester BD, Kaulius M, Ruissen GR, Dowd AJ and Beauchamp MR (2016) The effectiveness of multi-component goal setting interventions for changing physical activity behaviour: a systematic review and metaanalysis. Health Psychology Review 10(1): 67-88. 
Michie S, Atkins L and West R (2014) The behaviour change wheel. A guide to designing interventions. Great Britain: Silverback Publishing.

Michie, S, Van Stralen, MM, and West, R (2011) The behaviour change wheel: A new method for characterising and designing behaviour change interventions. Implementation Science 6(1): 42-53.

Moran R and Gutman LM (2021) Mental health training to improve communication with children and adolescents: A process evaluation. Journal of Clinical Nursing 30(3-4): 415-432.

Murray JM, Brennan SF, French DP, Patterson CC, Kee F and Hunter RF (2017) Effectiveness of physical activity interventions in achieving behaviour change maintenance in young and middle aged adults: a systematic review and meta-analysis. Social Science \& Medicine 192: 125-33.

Murtagh EM, Barnes AT, McMullen J and Morgan PJ (2018) Mothers and teenage daughters walking to health: using the behaviour change wheel to develop an intervention to improve adolescent girls' physical activity. Public Health 158:37-46.

O'Brien BC, Harris IB, Beckman TJ, Reed DA and Cook DA (2014) Standards for reporting qualitative research: A synthesis of recommendations. Academic Medicine 89(9): 1245-1251.

Rand M, Goyder E, Norman P, and Womack R (2020) Why do new members stop attending health and fitness venues? The importance of developing frequent and stable attendance behaviour. Psychology of Sport and Exercise 51: 101771.

Rebar AL, Stanton R, Geard D, Short C, Duncan MJ and Vandelanotte C (2015) A meta-metaanalysis of the effect of physical activity on depression and anxiety in non-clinical adult populations. Health Psychology Review 9(3): 366-78.

Rhodes RE, Janssen I, Bredin SS, Warburton DE and Bauman A (2017) Physical activity: Health impact, prevalence, correlates and interventions. Psychology and Health. 32(8): 942-75.

Rhodes RE and Pfaeffli LA (2010) Mediators of physical activity behaviour change among adult non-clinical populations: a review update. International Journal of Behavioral Nutrition and Physical Activity 7(1):1-1.

West, $R$ and Michie, S (2020) A brief introduction to the COM-B model of behaviour and the PRIME theory of motivation. Retrieved from https://www.qeios.com/read/WW04E6.2/pdf (accessed on 16 August 2021).

Williams DM, Papandonatos GD, Napolitano MA, Lewis BA, Whiteley JA and Marcus BH (2006) Perceived enjoyment moderates the efficacy of an individually tailored physical activity intervention. Journal of Sport and Exercise Psychology 28(3):300-9.

Williams SL and French DP (2011) What are the most effective intervention techniques for changing physical activity self-efficacy and physical activity behaviour-and are they the same? Health Education Research 26(2): 308-322.

World Health Organization (2010) Global recommendations on physical activity for health. Retrieved from https://www.who.int/dietphysicalactivity/global-PA-recs-2010.pdf (accessed on 09 August 2021). 
Table 1. Overview of participants and their characteristics.

\begin{tabular}{|c|c|c|c|c|c|}
\hline BC group & $\underline{\text { Gender }}$ & Age & $\begin{array}{l}\text { Interview } \\
\text { setting }\end{array}$ & Length $\mathrm{BC}$ membership & $\begin{array}{l}\text { Participant } \\
\text { number }\end{array}$ \\
\hline $\mathrm{BC} 1$ & $\mathrm{~F}$ & 39 & In-person & 3 years & P1 \\
\hline $\mathrm{BC} 1$ & $\mathrm{~F}$ & 55 & In-person & 5 years & P2 \\
\hline $\mathrm{BC} 1$ & $M$ & 50 & In-person & 2 years & P3 \\
\hline $\mathrm{BC} 1$ & $M$ & 28 & Phone & 18 months & P4 \\
\hline $\mathrm{BC} 1$ & $\mathrm{~F}$ & 33 & In-person & 16 months & P5 \\
\hline $\mathrm{BC} 1$ & $\mathrm{~F}$ & 33 & Phone & 2 years & P15 \\
\hline $\mathrm{BC} 2$ & $\mathrm{~F}$ & a & In-person & 13 months & P6 \\
\hline $\mathrm{BC} 2$ & $\mathrm{~F}$ & 63 & Skype & 20 months & P14 \\
\hline $\mathrm{BC} 3$ & $\mathrm{~F}$ & 51 & In-person & 6 years & P7 \\
\hline $\mathrm{BC} 3$ & $\mathrm{~F}$ & 52 & In-person & 2.5 years & P8 \\
\hline $\mathrm{BC} 3$ & $\mathrm{~F}$ & 44 & In-person & 3 years & P9 \\
\hline $\mathrm{BC} 3$ & $\mathrm{~F}$ & 55 & In-person & 7 years & P10 \\
\hline $\mathrm{BC} 3$ & $\mathrm{~F}$ & 58 & In-person & 6 years & P11 \\
\hline $\mathrm{BC} 3$ & $\mathrm{~F}$ & 63 & Skype & 5 years & P12 \\
\hline $\mathrm{BC} 3$ & $\mathrm{~F}$ & 50 & Skype & 1 year & P13 \\
\hline
\end{tabular}


Table 2. Identified facilitators and barriers to bootcamp participation.

\begin{tabular}{|c|c|c|}
\hline COM-B Domain & Sub-theme & Frequency \\
\hline Capability - Psychological & Exercise Knowledge (F) & 8 \\
\hline \multirow[t]{2}{*}{ Capability - Physical } & Physical Injury (B) & 8 \\
\hline & Physical Illness (B) & 5 \\
\hline \multirow[t]{8}{*}{ Opportunity - Physical } & Convenience (F) & 14 \\
\hline & Being Outdoors (F) & 10 \\
\hline & Variety of Activities (F) & 10 \\
\hline & Competing events (B) & 7 \\
\hline & Bad weather (B) & 6 \\
\hline & Upfront payment (F) & 6 \\
\hline & Travel (B) & 5 \\
\hline & Multiple Sessions (F) & 5 \\
\hline \multirow[t]{3}{*}{ Opportunity - Social } & Positive Instructor (F) & 14 \\
\hline & Group Members (F) & 11 \\
\hline & Supportive Partner (F) & 7 \\
\hline \multirow[t]{6}{*}{ Motivation - Reflective } & Health Benefits (F) & 14 \\
\hline & Weight Loss/Maintenance (F) & 10 \\
\hline & Accountability (F) & 8 \\
\hline & Prioritising exercise $(\mathrm{F})$ & 5 \\
\hline & Exercise Goals (F) & 2 \\
\hline & Social Identity (F) & 6 \\
\hline \multirow[t]{3}{*}{ Motivation - Automatic } & Enjoyment (F) & 13 \\
\hline & Feeling good (F) & 12 \\
\hline & Stress (B) & 3 \\
\hline
\end{tabular}

Note. Frequency refers to the number of participants (out of 15) who identified the reported barrier or facilitator. $(F)=$ Facilitator, $(B)=$ Barrier. 
Figure 1. The COM-B Model of Behaviour (West \& Michie, 2020).

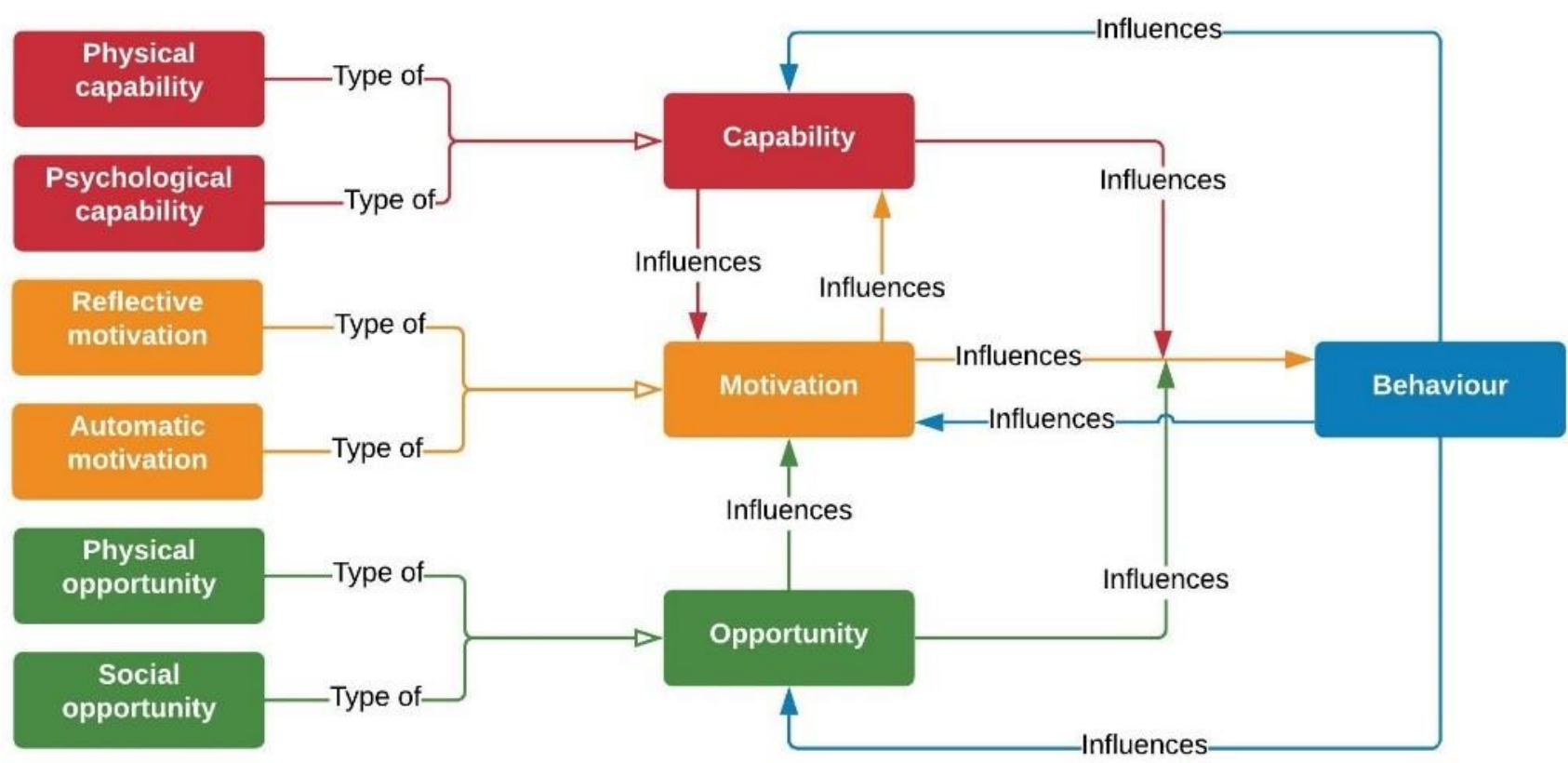

Capability is an attribute of a person that together with opportunity makes a behaviour possible or facilitates it.

Opportunity is an attribute of an environmental system that together with capability makes a behaviour possible or facilitates it.

Motivation is an aggregate of mental processes that energise and direct behaviour

Behaviour is individual human activity that involves co-ordinated contraction of striated muscles controlled by the brain.

Physical capability is capability that involves a person's physique, and musculoskeletal functioning (e.g. balance and dexterity)
Psychological capability is capability that involves a person's mental functioning (e.g. understanding and memory).

Reflective motivation is motivation that involves conscious thought processes (e.g. plans and evaluations)

Automatic motivation is motivation that involves habitual, instinctive, drive-related, and affective processes (e.g. desires and habits).

Physical opportunity is opportunity that involves inanimate parts of the environmental system and time (e.g. financial and mterial resources).

Social opportunity is opportunity that involves other people and organisations (e.g. culture and social norms). 